\title{
On inverses of some permutation polynomials over finite fields of characteristic three
}

\author{
Yanbin Zhenga,b,c, Fu Wang ${ }^{\mathrm{c}}$, Libo Wang ${ }^{\mathrm{d}}$, Wenhong Wei ${ }^{\mathrm{b}}$ \\ Corresponding author: Libo Wang, Email: wanglibo12b@mails.ucas.edu.cn \\ ${ }^{a}$ School of Mathematical Sciences, Qufu Normal University, Qufu, China \\ ${ }^{b}$ School of Computer Science and Technology, Dongguan University of Technology, Dongguan, China \\ ${ }^{c}$ Guangxi Key Laboratory of Cryptography and Information Security, Guilin University of Electronic \\ Technology, Guilin, China \\ ${ }^{d}$ College of Information Science and Technology, Jinan University, Guangzhou, China
}

\begin{abstract}
By using the piecewise method, Lagrange interpolation formula and Lucas' theorem, we determine explicit expressions of the inverses of a class of reversed Dickson permutation polynomials and some classes of generalized cyclotomic mapping permutation polynomials over finite fields of characteristic three.
\end{abstract}

Keywords: Permutation polynomials, Inverses, Reversed Dickson polynomials 2010 MSC: $11 \mathrm{~T} 06$

\section{Introduction}

For $q$ a prime power, let $\mathbb{F}_{q}$ denote the finite field with $q$ elements, $\mathbb{F}_{q}^{*}=\mathbb{F}_{q} \backslash\{0\}$, and $\mathbb{F}_{q}[x]$ the ring of polynomials over $\mathbb{F}_{q}$. A polynomial $f(x) \in \mathbb{F}_{q}[x]$ is called a permutation polynomial (PP) of $\mathbb{F}_{q}$ if it induces a bijection from $\mathbb{F}_{q}$ to itself. For any PP $f(x)$ of $\mathbb{F}_{q}$, there exists a polynomial $f^{-1}(x) \in \mathbb{F}_{q}[x]$ such that $f^{-1}(f(c))=c$ for each $c \in \mathbb{F}_{q}$ or equivalently $f^{-1}(f(x)) \equiv x$ $\left(\bmod x^{q}-x\right)$, and the polynomial $f^{-1}(x)$ is unique in the sense of reduction modulo $x^{q}-x$. Hence $f^{-1}(x)$ is defined as the composition inverse of $f(x)$, and we simply call it the inverse of $f(x)$ on $\mathbb{F}_{q}$.

Recently, some classes of PPs are found; see for example [18, 21, 32, 33, 35] for PPs of the form $x^{r} h\left(x^{q-1}\right)$ of $\mathbb{F}_{q^{2}},[24,37]$ for PPs of the form $\left(x^{q}-x+c\right)^{s}+L(x)$ of $\mathbb{F}_{q^{2}},[17,34,41]$ for PPs of the form $\left(a x^{q}+b x+c\right)^{r} \phi\left(\left(a x^{q}+b x+c\right)^{s}\right)+u x^{q}+v x$ of $\mathbb{F}_{q^{2}}$, [15] for PPs with low boomerang uniformity, and $[1,4,12]$ for PPs studied using the Hasse-Weil bound and Hermite's criterion. For a detailed introduction to the developments on PPs, we refer the reader to $[11,28]$ and the references therein.

The construction of PPs of finite fields is not an easy subject. However, the problem of determining the inverse of a PP seems to be an even more complicated problem. In fact, there are a few known classes of PPs whose inverses have been obtained explicitly; see for example [16, 19, 25, 39] for PPs of the form $x^{r} h\left(x^{(q-1) / d}\right)$, [30, 31] for linearized PPs, [27, 39] for generalized cyclotomic mapping PPs, [40] for general piecewise PPs, [3, 20, 36] for involutions over finite fields, and $[22,23]$ for more general classes of PPs. For a brief summary of the results concerning the inverses of PPs, we refer the reader to [38] and the references therein.

ฟhis paper has been published by ELSVIER available at https://doi.org/10.1016/j.ffa.2020.101670. Please refer to this paper as: Y. Zheng, F. Wang, L. Wang, W. Wei. On inverses of some permutation polynomials over finite fields of characteristic three. Finite Fields and Their Applications, 66:101670, 2020. 
The Dickson polynomial $D_{n}(x, a)$ of degree $n$ in the indeterminate $x$ and with parameter $a \in \mathbb{F}_{q}$ is given as

$$
D_{n}(x, a)=\sum_{i=0}^{\lfloor n / 2\rfloor} \frac{n}{n-i}\left(\begin{array}{c}
n-i \\
i
\end{array}\right)(-a)^{i} x^{n-2 i},
$$

where $\lfloor n / 2\rfloor$ denotes the largest integer $\leq n / 2$, and the term $\frac{n}{n-i}\left(\begin{array}{c}n-i \\ i\end{array}\right)$ is an integer. By reversing the roles of the indeterminate $x$ and the parameter $a$ in $D_{n}(x, a)$, the $n$-th reversed Dickson polynomials $D_{n}(a, x)$ was defined in [14] by

$$
D_{n}(a, x)=\sum_{i=0}^{\lfloor n / 2\rfloor} \frac{n}{n-i}\left(\begin{array}{c}
n-i \\
i
\end{array}\right)(-1)^{i} a^{n-2 i} x^{i}
$$

and their permutation properties were studied in [14]. Several families of reversed Dickson PPs over finite fields were given in $[10,14]$, which covered all the reversed Dickson PPs over $\mathbb{F}_{q}$ with $q<200$. Then, the notion of (reversed) Dickson polynomials of the $(k+1)$-th kind was introduced in [29], and the factorization and the permutation behavior of Dickson polynomials of the third kind were studied in [29]. Some necessary conditions for reversed Dickson polynomials of the first and second kinds to be PPs of finite fields were given in [13] and [9], respectively. The permutation behavior of reversed Dickson polynomials of the $(k+1)$-th kind was further investigated in [5-7]. In particular, Hou [10] proved the following result.

Lemma 1 ([10, Theorem1.1]). Let $n$ be a positive even integer. Then

$$
f(x)=\left(x-x^{2}-x^{3}\right) x^{\frac{3^{n}-1}{2}}-x+x^{2}
$$

is a PP of $\mathbb{F}_{3^{n}}$.

Since the reversed Dickson polynomial $D_{3^{n}+5}(1, x)=f(1-x)-1$, Hou equivalently proved that $D_{3^{n}+5}(1, x)$ is a PP of $\mathbb{F}_{3^{n}}$ for even $n$.

The purpose of this paper is to find the inverse of $f(x)$ in Lemma 1. The main idea is the combination of the piecewise method in [39] and some techniques in [38].

The rest of the paper is organized as follows. Section 2 gives a formula for the inverse of a class of piecewise PPs $f(x)$, which converts the problem of determining the inverse of $f(x)$ on $\mathbb{F}_{q}$ to the problem of computing the inverse $f_{s}^{-1}(x)$ of piece function $f_{s}(x)$ when restricted to a subset $C_{s}$ of $\mathbb{F}_{q}$ for all $s$. Then an expression of $f_{s}^{-1}(x)$ is presented in Theorem 2, which provides all the coefficients of $f_{s}^{-1}(x)$ by computing the coefficients of $x^{(q-3) / 2}$ and $x^{q-2}$ in $f_{s}(x)^{k}$ $\left(\bmod x^{q}-x\right)$ for $1 \leq k \leq(q-3) / 2$. By applying the results in Section 2 to $f(x)$ in Lemma 1, the coefficients of $f^{-1}(x)$ are reduced into two classes of binomial coefficients in Section 3. Section 4 gives explicit values of these binomial coefficients by using a congruence of binomial coefficients and Lucas' theorem. In other words, we determine the inverse of $f(x)$ as follows.

Theorem 1. The inverse of $f(x)$ in Lemma 1 on $\mathbb{F}_{3^{n}}$ is

$$
f^{-1}(x)=x^{3^{n-1}}\left(x^{\frac{3^{n}-1}{2}}+1\right)+\left(\sum_{j=0}^{n-1} \sum_{k=0}^{n-1}(-1)^{j+k} x^{\frac{3^{j}+3^{k}}{2}}\right)\left(x^{\frac{3^{n}-1}{2}}-1\right) .
$$

Furthermore, the inverse of $D_{3^{n}+5}(1, x)$ on $\mathbb{F}_{3^{n}}$ is $D_{3^{n}+5}^{-1}(1, x)=1-f^{-1}(x+1)$.

In the last section, by an argument similar to the one used in Theorem 1, we also obtain explicit inverses of some generalized cyclotomic mapping PPs studied in [26].

\section{The inverse of a class of piecewise PPs}

The piecewise methods for constructing PPs and their inverses were summarized in $[2,8]$ and [39] respectively. Applying these methods, we can easily get the following result. 
Lemma 2. Let $q$ be odd and $f_{0}(x), f_{1}(x) \in \mathbb{F}_{q}[x]$. Define

$$
f(x)=\frac{1}{2} f_{0}(x)\left(1+x^{\frac{q-1}{2}}\right)+\frac{1}{2} f_{1}(x)\left(1-x^{\frac{q-1}{2}}\right) \text { with } f(0)=0,
$$

$C_{0}=\left\{e^{2}: e \in \mathbb{F}_{q}^{*}\right\}$, and $C_{1}=\mathbb{F}_{q}^{*} \backslash C_{0}$. Then $f(x)$ is a PP of $\mathbb{F}_{q}$ if and only if $f_{s}$ is injective on $C_{s}$ and $0 \notin f_{s}\left(C_{s}\right)$ for $s \in\{0,1\}$, and $f_{0}\left(C_{0}\right) \cap f_{1}\left(C_{1}\right)=\emptyset$. Assume $f(x)$ is a PP of $\mathbb{F}_{q}$, and $f_{s}^{-1}(x) \in \mathbb{F}_{q}[x]$ satisfies that $f_{s}^{-1}(0)=0$ and $f_{s}^{-1}\left(f_{s}(c)\right)=c$ for any $c \in C_{s}$ and $s \in\{0,1\}$.

(i) If $f_{s}$ maps $C_{s}$ into $C_{s}$ for $s \in\{0,1\}$, then the inverse of $f(x)$ on $\mathbb{F}_{q}$ is

$$
f^{-1}(x)=\frac{1}{2} f_{0}^{-1}(x)\left(1+x^{\frac{q-1}{2}}\right)+\frac{1}{2} f_{1}^{-1}(x)\left(1-x^{\frac{q-1}{2}}\right) .
$$

(ii) If $f_{s}$ maps $C_{s}$ into $C_{t}$ for $s \neq t \in\{0,1\}$, then the inverse of $f(x)$ on $\mathbb{F}_{q}$ is

$$
f^{-1}(x)=\frac{1}{2} f_{0}^{-1}(x)\left(1-x^{\frac{q-1}{2}}\right)+\frac{1}{2} f_{1}^{-1}(x)\left(1+x^{\frac{q-1}{2}}\right) .
$$

Lemma 2 converts the problem of determining $f^{-1}(x)$ into the problem of computing $f_{s}^{-1}(x)$, the inverse of piece function $f_{s}(x)$ when restricted to $C_{s}$. In Lemma 2, if $q=3$, then $f(x)=$ $f^{-1}(x)= \pm x$ in the sense of reduction modulo $x^{3}-x$. We will give an expression of $f_{s}^{-1}(x)$ for $q>3$ after the following lemma.

Lemma 3. For $q$ an odd prime power, let $C_{0}=\left\{e^{2}: e \in \mathbb{F}_{q}^{*}\right\}$ and $C_{1}=\mathbb{F}_{q}^{*} \backslash C_{0}$. Then

$$
\sum_{a \in C_{s}} a^{k}=\left\{\begin{array}{cl}
-2^{-1} & \text { if } k=q-1, \\
(-1)^{s+1} 2^{-1} & \text { if } k=(q-1) / 2, \\
0 & \text { if } 1 \leq k \leq q-2 \text { and } k \neq(q-1) / 2 .
\end{array}\right.
$$

Proof. Let $A=\sum_{a \in C_{1}} a^{k}$ and $\xi$ a primitive element of $\mathbb{F}_{q}$. Obviously,

$$
\left(\xi^{k}+1\right) A=\sum_{a \in C_{0}} a^{k}+\sum_{a \in C_{1}} a^{k}=\sum_{a \in \mathbb{F}_{q}} a^{k}=\left\{\begin{aligned}
-1 & \text { if } k=q-1, \\
0 & \text { if } k=1,2, \ldots, q-2 .
\end{aligned}\right.
$$

If $k=q-1$, then $\xi^{k}=1$ and $2 A=-1$, so $A=-1 / 2$. If $k=(q-1) / 2$, then $a^{k}=-1$ for $a \in C_{1}$. Hence $A=(-1)(q-1) / 2=1 / 2$. If $1 \leq k \leq q-2$ and $k \neq(q-1) / 2$, then $\xi^{k} \neq-1$ and $\left(\xi^{k}+1\right) A=0$. Thus $A=0$. Then the result follows from (2).

Theorem 2. For an odd prime power $q>3$, let $C_{0}=\left\{e^{2}: e \in \mathbb{F}_{q}^{*}\right\}$ and $C_{1}=\mathbb{F}_{q}^{*} \backslash C_{0}$. For $s, t \in\{0,1\}$, assume $f_{s}(x) \in \mathbb{F}_{q}[x]$ induces a bijection from $C_{s}$ to $C_{t}$, and

$$
f_{s}(x)^{q-1-i} \equiv \sum_{0 \leq k \leq q-1} b_{i k} x^{k} \quad\left(\bmod x^{q}-x\right),
$$

where $(q+1) / 2 \leq i \leq q-2$. Then the inverse of $f_{s}(x)$ when restricted to $C_{s}$ is

$$
f_{s}^{-1}(x)=\sum_{(q+1) / 2 \leq i \leq q-2}(-1)^{s+t}\left(b_{i,(q-3) / 2}+(-1)^{s} b_{i, q-2}\right) x^{i-\frac{q-1}{2}}
$$

in the sense of reduction modulo $x^{\frac{q-1}{2}}+(-1)^{t+1}$.

Proof. Let $f_{s}^{-1}(x)=\sum_{i=0}^{q-1} c_{i} x^{i} \in \mathbb{F}_{q}[x]$. Then by the Lagrange interpolation formula,

$$
\begin{aligned}
f_{s}^{-1}(x) & =\sum_{a \in C_{s}} a\left(1-\left(x-f_{s}(a)\right)^{q-1}\right) \\
& =\sum_{a \in C_{s}} a\left(-\sum_{1 \leq i \leq q-1}(-1)^{i}\left(-f_{s}(a)\right)^{q-1-i} x^{i}\right) \\
& =\sum_{1 \leq i \leq q-1}\left(-\sum_{a \in C_{s}} a f_{s}(a)^{q-1-i}\right) x^{i} .
\end{aligned}
$$


Hence $c_{0}=0$ and

$$
c_{i}=-\sum_{a \in C_{s}} a f_{s}(a)^{q-1-i}, \quad \text { where } 1 \leq i \leq q-1 .
$$

Then by (3) and Lemma 3,

$$
\begin{aligned}
c_{i} & =-\sum_{a \in C_{s}} a \sum_{0 \leq k \leq q-1} b_{i k} a^{k}=-\sum_{0 \leq k \leq q-1} b_{i k} \sum_{a \in C_{s}} a^{k+1} \\
& =2^{-1}\left(b_{i, q-2}+(-1)^{s} b_{i,(q-3) / 2}\right) .
\end{aligned}
$$

We next reduce the degree of $f_{s}^{-1}(x)$. Since $f_{s}(x)$ induces a bijection from $C_{s}$ to $C_{t}$, we have $f_{s}(a) \in C_{t}$ and $f_{s}(a)^{(q-1) / 2}=(-1)^{t}$ for any $a \in C_{s}$, and so

$$
f_{s}(a)^{q-1-i}=(-1)^{t} f_{s}(a)^{q-1-i} / f_{s}(a)^{\frac{q-1}{2}}=(-1)^{t} f_{s}(a)^{q-1-\left(i+\frac{q-1}{2}\right)} .
$$

Substituting it into (5) yields

$$
c_{i}=(-1)^{t} c_{i+\frac{q-1}{2}}, \quad 1 \leq i \leq(q-1) / 2 .
$$

In particular, if $q>3$, then

$$
c_{(q-1) / 2}=(-1)^{t} c_{q-1}=(-1)^{t+1} \sum_{a \in C_{s}} a=0,
$$

where the last identity follows from Lemma 3. Therefore,

$$
\begin{aligned}
f_{s}^{-1}(x) & \stackrel{(8)}{=} \sum_{1 \leq i \leq(q-3) / 2}\left(c_{i} x^{i}+c_{i+\frac{q-1}{2}} x^{i+\frac{q-1}{2}}\right) \\
& \equiv \sum_{1 \leq i \leq(q-3) / 2}\left(c_{i}+(-1)^{t} c_{i+\frac{q-1}{2}}\right) x^{i} \quad\left(\bmod x^{\frac{q-1}{2}}+(-1)^{t+1}\right) \\
& \stackrel{(7)}{=} \sum_{1 \leq i \leq(q-3) / 2} 2(-1)^{t} c_{i+\frac{q-1}{2}} x^{i} \\
& =\sum_{(q+1) / 2 \leq i \leq q-2} 2(-1)^{t} c_{i} x^{i-\frac{q-1}{2}}
\end{aligned}
$$

Substituting (6) into (9) gives the desired result.

\section{The inverse of the $\mathbf{P P}$ in Lemma 1}

In this section, we will employ the results in Section 2 to compute the inverse of the PP $f(x)$ in Lemma 1. First, let $n>0$ be even, $C_{0}=\left\{e^{2}: e \in \mathbb{F}_{3^{n}}^{*}\right\}$ and $C_{1}=\mathbb{F}_{3^{n}}^{*} \backslash C_{0}$. Then $x^{\left(3^{n}-1\right) / 2}=1$ if $x \in C_{0}$, and $x^{\left(3^{n}-1\right) / 2}=-1$ if $x \in C_{1}$. Therefore,

$$
f(x)=\left(x-x^{2}-x^{3}\right) x^{\frac{3^{n}-1}{2}}-x+x^{2}
$$

can be written as

$$
f(x)= \begin{cases}0 & \text { if } x=0, \\ f_{0}(x):=-x^{3} & \text { if } x \in C_{0}, \\ f_{1}(x):=x(x+1)^{2} & \text { if } x \in C_{1} .\end{cases}
$$

Lemma 1 stated that $f(x)$ is a $\mathrm{PP}$ of $\mathbb{F}_{3^{n}}$. It means that $f_{0}(x)$ (resp. $f_{1}(x)$ ) induces an injection on $C_{0}$ (resp. $\left.C_{1}\right)$, and $f_{0}\left(C_{0}\right) \cap f_{1}\left(C_{1}\right)=\emptyset$. Since $n$ is even, $3^{n} \equiv 1(\bmod 4)$, and so $-1 \in C_{0}$. Hence $f_{0}(x)$ (resp. $\left.f_{1}(x)\right)$ induces a permutation on $C_{0}$ (resp. $C_{1}$ ). 
Since $\left(x^{3}\right)^{3^{n-1}}=x^{3^{n}}=x$ for any $x \in C_{0}$, the inverse of $f_{0}(x)$ on $C_{0}$ is

$$
f_{0}^{-1}(x)=-x^{3^{n-1}}
$$

We next apply Theorem 2 to determine the inverse of $f_{1}(x)$ on $C_{1}$. Denote $q=3^{n}, u=(q-3) / 2$, $v=q-2$ and $w_{i}=q-1-i$, where $(q+1) / 2 \leq i \leq q-2$. Then

$$
f_{1}(x)^{w_{i}}=x^{w_{i}}(x+1)^{2 w_{i}}=\sum_{0 \leq j \leq 2 w_{i}}\left(\begin{array}{c}
2 w_{i} \\
j
\end{array}\right) x^{w_{i}+j} .
$$

The degree of $f_{1}(x)^{w_{i}}$ is $3 w_{i}$, and $3 \leq 3 w_{i}<q-1+u$, as shown in Figure 1. Hence the coefficient

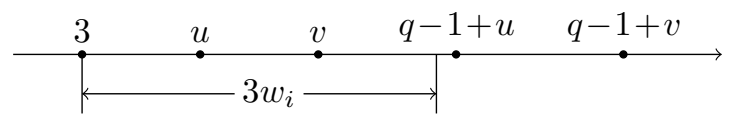

Figure 1: The range of $3 w_{i}$

of $x^{u}$ in (3) equals the coefficients of $x^{u}$ in (11), i.e., $b_{i u}=\left(\begin{array}{c}2 w_{i} \\ u-w_{i}\end{array}\right)$. Similarly, $b_{i v}=\left(\begin{array}{c}2 w_{i} \\ v-w_{i}\end{array}\right)$. If $3 w_{i}<u$, i.e., $i>(5 q-3) / 6$, then $b_{i u}=0$. If $3 w_{i}<v$, i.e., $i \geq 2 q / 3$, then $b_{i v}=0$. Hence we only need to consider the binomial coefficients

$$
\begin{aligned}
& b_{i u}=\left(\begin{array}{c}
2 q-2-2 i \\
i-\frac{q+1}{2}
\end{array}\right) \quad \text { for }(q+1) / 2 \leq i \leq(5 q-3) / 6, \\
& b_{i v}=\left(\begin{array}{c}
2 q-2-2 i \\
i-1
\end{array}\right) \quad \text { for }(q+1) / 2 \leq i<2 q / 3 .
\end{aligned}
$$

Lemma 4 ([38, Lemma 9$])$. Let $q$ be a prime $p$ power, and let $m, k$ be integers with $0 \leq k<q$. Then

$$
\left(\begin{array}{c}
q+m \\
k
\end{array}\right) \equiv\left(\begin{array}{c}
m \\
k
\end{array}\right) \quad(\bmod p)
$$

where $\left(\begin{array}{c}m \\ k\end{array}\right)=m(m-1) \cdots(m-k+1) / k$ !

Employing Lemma 4 and the fact $\left(\begin{array}{c}-m \\ k\end{array}\right)=(-1)^{k}\left(\begin{array}{c}m+k-1 \\ k\end{array}\right)$ for $m, k>0$, we have

$$
b_{i v} \equiv\left(\begin{array}{c}
-2-2 i \\
i-1
\end{array}\right) \equiv(-1)^{i-1}\left(\begin{array}{c}
3 i \\
i-1
\end{array}\right) \quad(\bmod 3),
$$

where $(q+1) / 2 \leq i<2 q / 3$. Similarly, if $(q+1) / 2 \leq i \leq(5 q-3) / 6$, then

$$
b_{i u} \equiv\left(\begin{array}{c}
-2-2 i \\
i-\frac{q+1}{2}
\end{array}\right) \equiv(-1)^{i-\frac{q+1}{2}}\left(\begin{array}{c}
3 i-\frac{q-1}{2} \\
i-\frac{q+1}{2}
\end{array}\right) \quad(\bmod 3) .
$$

By Lemma 2, Theorem 2, (10), (12) and (13), the inverse of $f(x)$ in Lemma 1 on $\mathbb{F}_{3^{n}}$ is

$$
f^{-1}(x)=x^{3^{n-1}}\left(x^{\frac{3^{n}-1}{2}}+1\right)+f_{1}^{-1}(x)\left(x^{\frac{3^{n}-1}{2}}-1\right),
$$

where

$$
\begin{aligned}
f_{1}^{-1}(x)= & \sum_{\left(3^{n}+1\right) / 2 \leq i \leq\left(5 \cdot 3^{n}-3\right) / 6}(-1)^{i-\frac{3^{n}+1}{2}}\left(\begin{array}{c}
3 i-\frac{3^{n}-1}{2} \\
i-\frac{3^{n}+1}{2}
\end{array}\right) x^{i-\frac{3^{n}-1}{2}} \\
& +\sum_{\left(3^{n}+1\right) / 2 \leq i \leq 2 \cdot 3^{n} / 3}(-1)^{i}\left(\begin{array}{c}
3 i \\
i-1
\end{array}\right) x^{i-\frac{3^{n}-1}{2}}
\end{aligned}
$$

in the sense of reduction modulo $x^{\frac{3^{n}-1}{2}}+1$. 


\section{Explicit values of binomial coefficients}

This section will give the explicit values of binomial coefficients in (15).

Theorem 3. Let $1 \leq i<3^{n}$ with $n \geq 1$. Then $\left(\begin{array}{c}3 i \\ i-1\end{array}\right) \not \equiv 0(\bmod 3)$ if and only if $i=\left(3^{k}-1\right) / 2$, where $k=1,2, \ldots, n$.

Proof. If $1 \leq i<3^{n}$, then we can write $i=i_{0}+i_{1} 3+i_{2} 3^{2}+\cdots+i_{n-1} 3^{n-1}$, where $i_{t}=0,1,2$ for $0 \leq t \leq n-1$. Then

$$
\begin{aligned}
3 i & =0+i_{0} 3+i_{1} 3^{2}+\cdots+i_{n-2} 3^{n-1}+i_{n-1} 3^{n}, \\
i-1 & =i_{0}-1+i_{1} 3+i_{2} 3^{2}+\cdots+i_{n-1} 3^{n-1} .
\end{aligned}
$$

By Lucas' theorem, if $i_{0}=0$, then $\left(\begin{array}{c}3 i \\ i-1\end{array}\right) \equiv\left(\begin{array}{l}0 \\ 2\end{array}\right) \equiv 0(\bmod 3)$, and if $i_{0}=1,2$, then

$$
\left(\begin{array}{c}
3 i \\
i-1
\end{array}\right) \equiv\left(\begin{array}{c}
0 \\
i_{0}-1
\end{array}\right)\left(\begin{array}{c}
i_{0} \\
i_{1}
\end{array}\right)\left(\begin{array}{c}
i_{1} \\
i_{2}
\end{array}\right) \cdots\left(\begin{array}{c}
i_{n-2} \\
i_{n-1}
\end{array}\right)\left(\begin{array}{c}
i_{n-1} \\
0
\end{array}\right) \quad(\bmod 3)
$$

Hence $\left(\begin{array}{c}3 i \\ i-1\end{array}\right) \not \equiv 0(\bmod 3)$ if and only if one of the following conditions holds:

1. $i_{0}=1$ and $1 \geq i_{1} \geq i_{2} \geq \cdots \geq i_{n-2} \geq i_{n-1} \geq 0$;

2. $i=1,1+3,1+3+3^{2}, \ldots, 1+3+3^{2}+\cdots+3^{n-1}$;

3. $i=\left(3^{k}-1\right) / 2$, where $k=1,2, \ldots, n$.

Corollary 1. Let $\left(3^{n}-1\right) / 2<i<3^{n}$ with $n \geq 1$. Then $\left(\begin{array}{c}3 i \\ i-1\end{array}\right) \equiv 0(\bmod 3)$.

It is easy to obtain the following 3 -adic expansion:

$$
\left(5 \cdot 3^{n}-3\right) / 6-\left(3^{n}+1\right) / 2=2+2 \cdot 3+2 \cdot 3^{2}+\cdots+2 \cdot 3^{n-2} .
$$

Thus, when $\left(3^{n}+1\right) / 2 \leq i \leq\left(5 \cdot 3^{n}-3\right) / 6$, we can write

$$
i=\left(3^{n}+1\right) / 2+i_{0}+i_{1} \cdot 3+i_{2} \cdot 3^{2}+\cdots+i_{n-2} \cdot 3^{n-2},
$$

where $i_{t}=0,1,2$ for $0 \leq t \leq n-2$. Then by an argument similar to the one used in [38, Theorems 11 and 12], we obtain the following two results.

Theorem 4. Let $\left(3^{n}+1\right) / 2 \leq i \leq\left(5 \cdot 3^{n}-3\right) / 6$ with $n \geq 1$. Then the following statements are equivalent:

(i) $\left(\begin{array}{c}3 i-\frac{3^{n}-1}{2} \\ i-\frac{3^{n}+1}{2}\end{array}\right) \not \equiv 0(\bmod 3) ;$

(ii) $2 \geq i_{0} \geq i_{1} \geq \cdots \geq i_{n-2} \geq 0$, where $i_{0}, i_{1}, \ldots, i_{n-2}$ are defined by (16);

(iii) $i=\frac{3^{n}+1}{2}+\frac{3^{j}-1}{2}+\frac{3^{k}-1}{2}$, where $0 \leq j \leq k \leq n-1$.

Theorem 5. Let $i=\frac{3^{n}+1}{2}+\frac{3^{j}-1}{2}+\frac{3^{k}-1}{2}$, where $0 \leq j \leq k \leq n-1$. Then

$$
\left(\begin{array}{c}
3 i-\frac{3^{n}-1}{2} \\
i-\frac{3^{n}+1}{2}
\end{array}\right) \equiv\left\{\begin{array}{lll}
1 & (\bmod 3) & \text { if } j=k, \\
2 & (\bmod 3) & \text { if } j<k .
\end{array}\right.
$$

According to Theorem 5, we obtain the following result. 
Theorem 6. Let $i=\frac{3^{n}+1}{2}+\frac{3^{j}-1}{2}+\frac{3^{k}-1}{2}$, where $0 \leq j \leq k \leq n-1$. Then

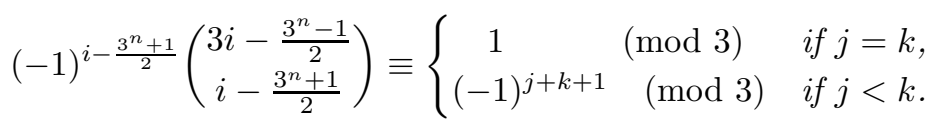

Proof. Since $3^{m}=(1+2)^{m}=1+\left(\begin{array}{c}m \\ 1\end{array}\right) 2+\left(\begin{array}{c}m \\ 2\end{array}\right) 2^{2}+\cdots+\left(\begin{array}{c}m \\ m\end{array}\right) 2^{m}$, we have $(-1)^{\frac{3^{m}-1}{2}}=(-1)^{m}$, and so $(-1)^{i-\frac{3^{n}+1}{2}}=(-1)^{\frac{3^{j}-1}{2}+\frac{3^{k}-1}{2}}=(-1)^{j+k}$. Substituting it into Theorem 5 gives the desired result.

By Corollary 1, Theorems 4 and 6, we can write (15) as the following form:

$$
\begin{aligned}
f_{1}^{-1}(x) & =\sum_{0 \leq k \leq n-1} x^{3^{k}}+\sum_{0 \leq j<k \leq n-1} 2(-1)^{j+k} x^{\frac{3^{j}+3^{k}}{2}} \\
& =\sum_{0 \leq j=k \leq n-1}(-1)^{j+k} x^{\frac{3^{j}+3^{k}}{2}}+\sum_{0 \leq j \neq k \leq n-1}(-1)^{j+k} x^{\frac{3^{j}+3^{k}}{2}} \\
& =\sum_{0 \leq j, k \leq n-1}(-1)^{j+k} x^{\frac{3^{j}+3^{k}}{2}} .
\end{aligned}
$$

Substituting (17) into (14) completes the proof of Theorem 1.

\section{Slight generalization}

In this section, we also let $C_{0}=\left\{e^{2}: e \in \mathbb{F}_{3^{n}}^{*}\right\}$ and $C_{1}=\mathbb{F}_{3^{n}}^{*} \backslash C_{0}$. Let $\eta$ be the quadratic character. By an argument similar to that used in Theorem 1, we deduce the inverses of some generalized PPs studied in [26].

Lemma 5 ([26, Theorem 4.7]). Let $\alpha, \beta, \gamma, \theta \in \mathbb{F}_{3^{n}}^{*}$ with $n \geq 1$, and let

$$
f(x)= \begin{cases}0 & \text { if } x=0, \\ \alpha\left(x^{3}+\gamma x^{2}+\gamma^{2} x\right) & \text { if } x \in C_{0}, \\ \beta\left(x^{3}+\theta x^{2}+\theta^{2} x\right) & \text { if } x \in C_{1} .\end{cases}
$$

Then $f(x)$ is a PP of $\mathbb{F}_{3^{n}}$ if and only if $\eta(\gamma)=-1, \eta(\theta)=1$ and $\eta(\alpha)=\eta(\beta)$.

Lemma 1 is a special case of Lemma 5 for $n$ is even, $\gamma=0$ and $\alpha=-\beta=\theta=-1$. The following result gives the inverse of $f(x)$ in Lemma 5.

Theorem 7. If $f(x)$ in Lemma 5 is a PP of $\mathbb{F}_{3^{n}}$ and $\eta(\alpha)=(-1)^{m}$ with $m \in\{0,1\}$, then

$$
f^{-1}(x)=-u(x)\left(1+(-1)^{m} x^{\left(3^{n}-1\right) / 2}\right)-v(x)\left(1+(-1)^{m+1} x^{\left(3^{n}-1\right) / 2}\right),
$$

where

$$
\begin{aligned}
& u(x)=\sum_{0 \leq j, k \leq n-1} \gamma\left(\alpha^{-1} \gamma^{-3} x\right)^{\frac{3^{j}+3^{k}}{2}}, \\
& v(x)=\sum_{0 \leq j, k \leq n-1} \theta\left(\beta^{-1} \theta^{-3} x\right)^{\frac{3^{j}+3^{k}}{2}} .
\end{aligned}
$$

Proof. Let $\tau, \lambda \in \mathbb{F}_{3^{n}}^{*}, s \in\{0,1\}$ and $f_{s}(x)=\tau\left(x^{3}+\lambda x^{2}+\lambda^{2} x\right)=\tau x(x-\lambda)^{2}$. If $f(x)$ in Lemma 5 is a $\mathrm{PP}$ of $\mathbb{F}_{3^{n}}$ and $\eta(\tau)=(-1)^{m}$ with $m \in\{0,1\}$, then $f_{s}(x)$ induces a bijection from $C_{s}$ to $C_{t}$, where $s, t \in\{0,1\}$. Clearly, $s=t$ if $m=0$, and $s \neq t$ if $m=1$. Hence $s+t+m \equiv 0(\bmod 2)$. Let $w_{i}=3^{n}-1-i$, where $\left(3^{n}+1\right) / 2 \leq i \leq 3^{n}-2$. Then

$$
f_{s}(x)^{w_{i}}=(\tau x)^{w_{i}}(x-\lambda)^{2 w_{i}}=\sum_{0 \leq j \leq 2 w_{i}} \tau^{w_{i}}(-\lambda)^{2 w_{i}-j}\left(\begin{array}{c}
2 w_{i} \\
j
\end{array}\right) x^{w_{i}+j}
$$


By an argument similar to that used in the previous sections, we have

$$
\begin{aligned}
f_{s}^{-1}(x)= & (-1)^{s+t}\left(\sum_{0 \leq k \leq n-1} \tau^{\frac{3^{n}-1}{2}-3^{k}} \lambda^{3^{n}-3^{k+1}} x^{3^{k}}\right. \\
& \left.+\sum_{0 \leq j<k \leq n-1} 2 \tau^{\frac{3^{n}-1}{2}-\frac{3^{j}+3^{k}}{2}} \lambda^{3^{n}-3 \frac{3^{j}+3^{k}}{2}} x^{\frac{3^{j}+3^{k}}{2}}\right) \\
= & (-1)^{s+t} \tau^{\frac{3^{n}-1}{2}} \sum_{0 \leq j, k \leq n-1} \lambda^{3^{n}}\left(\tau^{-1} \lambda^{-3} x\right)^{\frac{3^{j}+3^{k}}{2}} \\
= & (-1)^{s+t+m} \sum_{0 \leq j, k \leq n-1} \lambda\left(\tau^{-1} \lambda^{-3} x\right)^{\frac{3^{j}+3^{k}}{2}} \\
= & \sum_{0 \leq j, k \leq n-1} \lambda\left(\tau^{-1} \lambda^{-3} x\right)^{\frac{3^{j}+3^{k}}{2}} .
\end{aligned}
$$

Substituting it into Lemma 2 gives the desired result.

Lemma 6 ([26, Theorem 4.2]). Let $\alpha, \beta, \theta \in \mathbb{F}_{3^{n}}^{*}$ with $n \geq 1$, and let

$$
f(x)= \begin{cases}0 & \text { if } x=0, \\ \alpha x^{t} & \text { if } x \in C_{0}, \\ \beta\left(x^{3}+\theta x^{2}+\theta^{2} x\right) & \text { if } x \in C_{1} .\end{cases}
$$

Then $f(x)$ is a PP of $\mathbb{F}_{3^{n}}$ if and only if $\left(t, \frac{3^{n}-1}{2}\right)=1, \eta(\theta)=1$ and $\eta(\alpha)=\eta(\beta)$.

Theorem 8. If $f(x)$ in Lemma 6 is a PP of $\mathbb{F}_{3^{n}}$ and $\eta(\alpha)=(-1)^{m}$ with $m \in\{0,1\}$, then

$$
f^{-1}(x)=-u(x)\left(1+(-1)^{m} x^{\left(3^{n}-1\right) / 2}\right)-v(x)\left(1+(-1)^{m+1} x^{\left(3^{n}-1\right) / 2}\right),
$$

where $v(x)$ is defined by $(20), u(x)=\left(\alpha^{-1} x\right)^{s}$, and $s$ is the inverse of $t$ modulo $\left(3^{n}-1\right) / 2$.

Lemma 7 ([26, Corollary 4.5]). Let $n, t$ be positive integers. Let $\alpha, \beta, \gamma \in \mathbb{F}_{3^{n}}^{*}$ and

$$
f(x)= \begin{cases}0 & \text { if } x=0, \\ \alpha\left(x^{3}+\gamma x^{2}+\gamma^{2} x\right) & \text { if } x \in C_{0}, \\ \beta x^{t} & \text { if } x \in C_{1} .\end{cases}
$$

Then $f(x)$ is a PP of $\mathbb{F}_{3^{n}}$ if and only if $\left(t, \frac{3^{n}-1}{2}\right)=1, \eta(\gamma)=-1$ and $\eta(\alpha)=\eta(\beta)(-1)^{t+1}$.

Theorem 9. If $f(x)$ in Lemma 7 is a PP of $\mathbb{F}_{3^{n}}$ and $\eta(\alpha)=(-1)^{m}$ with $m \in\{0,1\}$, then

$$
f^{-1}(x)=-u(x)\left(1+(-1)^{m} x^{\left(3^{n}-1\right) / 2}\right)-v(x)\left(1+(-1)^{m+1} x^{\left(3^{n}-1\right) / 2}\right),
$$

where $u(x)$ is defined by $(19), v(x)=(-1)^{r}\left(\beta^{-1} x\right)^{s}, r$ and $s$ are integers such that $1 \leq s<$ $\left(3^{n}-1\right) / 2$ and $s t+r\left(3^{n}-1\right) / 2=1$.

When $n=1$, it is easy to verify that $f(x) \equiv f^{-1}(x) \equiv \pm x\left(\bmod x^{3}-x\right)$ in all results of this section. Hence Theorems 7, 8 and 9 are all true for $n=1$. If $\gamma=\theta=0$, then Lemmas 5,6 and 7 are the special cases of [26, Corollary 2.3], and their inverses are given in [27, 39].

\section{Acknowledgments}

We are grateful to the referees for many useful comments and suggestions. 


\section{References}

[1] D. Bartoli, On a conjecture about a class of permutation trinomials, Finite Fields Appl. 52 (2018) 30-50.

[2] X. Cao, L. Hu, Z. Zha, Constructing permutation polynomials from piecewise permutations, Finite Fields Appl. 26 (2014) 162-174.

[3] P. Charpin, S. Mesnager, S. Sarkar, Involutions over the Galois Field $\mathbb{F}_{2^{n}}$, IEEE Trans. Inf. Theory 62 (4) (2016) 2266-2276.

[4] W.-S. Chou, X.-D. Hou, On a conjecture of Fernando, Hou and Lappano concerning permutation polynomials over finite fields, Finite Fields Appl. 56 (2019) 58-92.

[5] N. Fernando, Reversed Dickson polynomials of the $(k+1)$-th kind over finite fields, J. Number Theory 172 (2017) 234-255, doi:10.1016/j.jnt.2016.08.018.

[6] N. Fernando, A note on permutation binomials and trinomials over finite fields, N. Z. J. Math. 48 (2018) 25-29.

[7] N. Fernando, Reversed Dickson polynomials of the $(k+1)$-th kind over finite fields, II, arXiv:1706.01391v3, https://arxiv.org/abs/1706.01391, 2018.

[8] N. Fernando, X.-D. Hou, A piecewise construction of permutation polynomials over finite fields, Finite Fields Appl. 18 (6) (2012) 1184-1194.

[9] S. Hong, X. Qin, W. Zhao, Necessary conditions for reversed Dickson polynomials of the second kind to be permutational, Finite Fields Appl. 37 (2016) 54-71.

[10] X.-D. Hou, Two classes of permutation polynomials over finite fields, J. Comb. Theory Ser. A 118 (2011) 448-454.

[11] X.-D. Hou, Permutation polynomials over finite fields - a survey of recent advances, Finite Fields Appl. 32 (2015) 82-119.

[12] X.-D. Hou, Applications of the Hasse-Weil bound to permutation polynomials, Finite Fields Appl. 54 (2018) 113-132.

[13] X.-D. Hou, T. Ly, Necessary conditions for reversed Dickson polynomials to be permutational, Finite Fields Appl. 16 (2010) 436-448.

[14] X.-D. Hou, G. L. Mullen, J. A. Sellers, J. L. Yucas, Reversed Dickson polynomials over finite fields, Finite Fields Appl. 15 (2009) 748-773.

[15] K. Li, L. Qu, B. Sun, C. Li, New results about the Boomerang uniformity of permutation polynomials, IEEE Trans. Inf. Theory 65 (11) (2019) 7542-7553, doi:10.1109/TIT.2019.2918531.

[16] K. Li, L. Qu, Q. Wang, Compositional inverses of permutation polynomials of the form $x^{r} h\left(x^{s}\right)$ over finite fields, Cryptogr. Commun. 11 (2019) 279-298.

[17] L. Li, S. Wang, C. Li, X. Zeng, Permutation polynomials $\left(x^{p^{m}}-x+\delta\right)^{s_{1}}+\left(x^{p^{m}}-x+\delta\right)^{s_{2}}+x$ over $\mathbb{F}_{p^{n}}$, Finite Fields Appl. 51 (2018) 31-61.

[18] N. Li, On two conjectures about permutation trinomials over $\mathbb{F}_{32 k}$, Finite Fields Appl. 47 (2017) $1-10$.

[19] A. Muratović-Ribić, A note on the coefficients of inverse polynomials, Finite Fields Appl. 13 (2007) 977-980.

[20] T. Niu, K. Li, L. Qu, Q. Wang, New constructions of involutions over finite fields, Cryptogr. Commun. 12 (2020) 165-185, doi:10.1007/s12095-019-00386-2.

[21] Z. Tu, X. Zeng, Two classes of permutation trinomials with Niho exponents, Finite Fields Appl. 53 (2018) 99-112.

[22] A. Tuxanidy, Q. Wang, On the inverses of some classes of permutations of finite fields, Finite Fields Appl. 28 (2014) 244-281.

[23] A. Tuxanidy, Q. Wang, Compositional inverses and complete mappings over finite fields, Discrete Appl. Math. 217 (2017) 318-329.

[24] L. Wang, B. Wu, General constructions of permutation polynomials of the form $\left(x^{2^{m}}+x+\right.$ $\delta)^{i\left(2^{m}-1\right)+1}+x$ over $\mathbb{F}_{2^{2 m}}$, Finite Fields Appl. 52 (2018) 137-155.

[25] Q. Wang, On inverse permutation polynomials, Finite Fields Appl. 15 (2009) 207-213.

[26] Q. Wang, Cyclotomy and permutation polynomials of large indices, Finite Fields Appl. 22 (2013) 57-69.

[27] Q. Wang, A note on inverses of cyclotomic mapping permutation polynomials over finite fields, Finite Fields Appl. 45 (2017) 422-427. 
[28] Q. Wang, Polynomials over finite fields: an index approach, in: K.-U. Schmidt, A. Winterhof (Eds.), Combinatorics and Finite Fields: Difference Sets, Polynomials, Pseudorandomness and Applications, 319-346,2019, doi:10.1515/9783110642094-015.

[29] Q. Wang, J. L. Yucas, Dickson polynomials over finite fields, Finite Fields Appl. 18 (2012) 814-831.

[30] B. Wu, The compositional inverses of linearized permutation binomials over finite fields, arXiv:1311.2154v1, https://arxiv.org/abs/1311.2154, 2013.

[31] B. Wu, Z. Liu, Linearized polynomials over finite fields revisited, Finite Fields Appl. 22 (2013) 79-100.

[32] D. Wu, P. Yuan, C. Ding, Y. Ma, Permutation trinomials over $\mathbb{F}_{2^{m}}$, Finite Fields Appl. 46 (2017) $38-56$.

[33] G. Xu, X. Cao, J. Ping, Some permutation pentanomials over finite fields with even characteristic, Finite Fields Appl. 49 (2018) 212-226.

[34] X. Xu, X. Feng, X. Zeng, Complete permutation polynomials with the form $\left(x^{p^{m}}-x+\delta\right)^{s}+a x^{p^{m}}+b x$ over $\mathbb{F}_{p^{n}}$, Finite Fields Appl. 57 (2019) 309-343, doi:10.1016/j.ffa.2019.03.001.

[35] Z. Zha, L. Hu, S. Fan, Further results on permutation trinomials over finite fields with even characteristic, Finite Fields Appl. 45 (2017) 43-52.

[36] D. Zheng, M. Yuan, N. Li, L. Hu, X. Zeng, Constructions of involutions over finite fields, IEEE Trans. Inf. Theory 65 (12) (2019) 7876-7883, doi:10.1109/TIT.2019.2919511.

[37] D. Zheng, M. Yuan, L. Yu, Two types of permutation polynomials with special forms, Finite Fields Appl. 56 (2019) 1-16.

[38] Y. Zheng, Q. Wang, W. Wei, On inverses of permutation polynomials of small degree over finite fields, IEEE Trans. Inf. Theory 66 (2) (2020) 914-922, doi:10.1109/TIT.2019.2939113.

[39] Y. Zheng, Y. Yu, Y. Zhang, D. Pei, Piecewise constructions of inverses of cyclotomic mapping permutation polynomials, Finite Fields Appl. 40 (2016) 1-9.

[40] Y. Zheng, P. Yuan, D. Pei, Piecewise constructions of inverses of some permutation polynomials, Finite Fields Appl. 36 (2015) 151-169.

[41] Y. Zheng, P. Yuan, D. Pei, Large classes of permutation polynomials over $\mathbb{F}_{q^{2}}$, Des. Codes Cryptogr. 81 (2016) 505-521. 